\title{
Trabajo Social en Suecia, tendencias generales y el caso particular de los niños migrantes
}

\author{
Social Work in Sweden, general trends and the particular case \\ of migrant children
}

\author{
Norma MONTESINO \\ Universidad de Lund (Suecia) \\ norma.montesino@soch.lu.es \\ Erica RIGHARD \\ Universidad de Malmö (Suecia) \\ erica.righard@mah.se
}

Recibido: 01/08/2013

Revisado: 11/09/2013

Aceptado: 28/02/2014

Disponible on line: $27 / 06 / 2014$

\begin{abstract}
Resumen
La reestructuración del estado de bienestar sueco y el aumento de las desigualdades son transformaciones sociales ocurridas durante los tres últimos decenios. Suecia ha perdido su posición de liderazgo en los rankings internacionales sobre igualdad social. Nuevas formas de entender y abordar los problemas sociales permiten la privatización de los servicios sociales antes públicos. Además, cambios demográficos como el aumento de la movilidad internacional y la complejidad en la dinámica de los problemas sociales exigen nuevas respuestas de los servicios sociales. Esto tiene claras implicaciones para el Trabajo Social que surgió como una actividad delimitada territorialmente por las fronteras nacionales. El caso de los niños migrantes es un ejemplo ya que expresa claramente esas nuevas problemáticas y muestra diversas y contradictorias respuestas desde los servicios de protección de la infancia. En este artículo discutimos las respuestas que dan los trabajadores sociales en la atención de casos y las respuestas institucionalizadas desde los Hogares de acogida. En esos lugares detectamos el fracaso de las formas institucionalizadas de protección de la infancia. En la atención de casos individuales vemos surgir respuestas que, dentro de los marcos de la legalidad, cuestionan una tradición de lealtad y subordinación a la autoridad en la historia del Trabajo Social en Suecia. Aparte de esas respuestas vemos también surgir organizaciones que cuestionan políticas represivas e intentan trabajar desde una perspectiva que toma en cuenta las problemáticas que afectan a los niños migrantes.
\end{abstract}

Palabras claves: Trabajo Social, Suecia, estado de bienestar, niños migrantes, protección de la infancia.

\begin{abstract}
Ongoing restructuring of the Swedish welfare state and increased social cleavages in the population are interlinked with social transformations that go back about three decades in time. While Sweden used to hold top positions in international rankings of social equality, today it typically only reaches to mediocre rankings. Shifting understandings and ways of addressing social problems have opened up for a marketization of previously public social services. In addition, demographic changes such as increased international mobility and the complexity of cross-border dynamics of social problems are demanding new responses from the social services. This means that as social work emerged as an activity territorially bounded by national borders, it is now challenged by globalization processes at multiple societal levels. We shall here take up the case of migrant children as an illustrative case.

In Sweden migrant children are perceived as a new area of social intervention, to which the child protection services respond in diverse and contradictory ways. We discuss the individual responses by social workers and the institutional responses from homes of residential care for children. In the individual case-work we see an emergence of critical responses among social workers, who within the legal framework question the tradition of loyalty to Swedish state authority in social work. By contrast, the institutional responses seem to violate instead of protecting migrant children. Apart from these responses, we describe the emergence of new organizations that challenge repressive migration policies and which seek to work from the perspective of the migrant children.
\end{abstract}

Keywords: social work, Sweden, welfare state, migrant children, child protection.

Referencia normalizada: Montesino, N. y Righard, E. (2014: «Trabajo Social en Suecia, tendencias generales y el caso particular de los niños migrantes». Cuadernos de Trabajo Social, 27 (1): 39-48.

Sumario: Introducción. 1. Un Estado de bienestar en proceso de transformación. 2. Trabajo Social, infancia y niños migrantes. 3. Atención individual. 4. Hogares de acogida. 5. Conclusiones. 6. Referencias bibliográficas. 


\section{Introducción}

Trabajo Social es un área de intervención que surgió en Suecia hace poco más de un siglo; las actividades de esta área siempre se han desarrollado dentro de los marcos territoriales del estado nacional. Individuos o grupos, incluidos en la estadística que por razones sociales (edad, situación familiar, etc.) o de salud (invalidez o enfermedad) no están en condiciones de trabajar, han sido y son objeto de las intervenciones sociales. El Trabajo Social está formalmente organizado en los Servicios de Bienestar Social que están divididos en diferentes áreas: asistencia económica, infancia y familia, dependencia de drogas y atención tanto de ancianos como de personas con algún tipo de discapacidad. La mayor parte de los trabajadores sociales trabajan dentro de las áreas de asistencia económica, infancia y familia.

El estado sueco ha jugado siempre un papel central en el Trabajo Social que se refleja tanto en la organización de los sistemas de bienestar social como en la estructura de empleo de quienes trabajan en esta área. Los profesionales del Trabajo Social son en su mayoría funcionarios de la esfera pública (estado y municipios). El estado ha delegado el ejercicio del bienestar social a los municipios, quienes emplean a la mayoría de los trabajadores sociales. Esta estructura de empleo se mantiene aun cuando aumentan las privatizaciones (a menudo subvencionadas por el estado o los municipios). La delegación del poder estatal a los municipios está regulada por la Ley de servicios sociales, ley que define la mayor parte de los objetivos dejando un espacio discrecional moderado para el ejercicio profesional.

Desde el año 1977, el Trabajo Social es una disciplina académica integrada a la estructura organizativa de las universidades suecas. El título de Trabajo Social se obtiene después de tres años y medio de estudios universitarios, dentro de un programa educacional formalizado de acuerdo a directivas formuladas por la autoridad estatal de educación superior. La formación universitaria incluye también estudios de maestría y doctorado en Trabajo Social (Sunesson, 2003). El Trabajo Social es entonces una disciplina académica independiente con sus propias áreas de investigación. Este proceso de academización ha contribuido al fortalecimiento de la profesión, un proceso debilitado últimamente por lo que algunos denominan desprofesionalización, es decir un cuestionamiento de la autonomía profesional creado por nuevas formas de abordar los problemas sociales denominada la Nueva Gestión Social (NGS). La NGS legitima la reestructuración de los Servicios de Bienestar Social en los países europeos y crea diferentes respuestas de acuerdo a las peculiaridades históricas de cada estado de bienestar (Ellison, 2007; Lorenz, 2006). Existen diferentes interpretaciones sobre las consecuencias de estos procesos expresadas también en divergencias sobre cuáles son los conocimientos que deben usarse para intervenir en los problemas sociales (Bergmark, 2012; Dellgran, 2012). Pensamos que estas discrepancias también deberían incluir una reflexión sobre las premisas que han condicionado los contenidos del conocimiento en nuestra disciplina.

En este artículo discutimos tendencias generales de los procesos de transformación del estado de bienestar en Suecia. Primero describimos las características generales de la transformación del estado de bienestar, para después discutir el significado de esos procesos en el área del Trabajo Social. Las formas de organización y las posibilidades de acción de los profesionales las ilustramos desde ciertas prácticas de Trabajo Social con niños migrantes que llegan solos a Suecia. La llegada de estos niños cuestiona representaciones internalizadas sobre lo que constituye la normalidad, representaciones que incluyen concepciones fundamentales sobre la infancia, el rol de la familia y los límites de los derechos ciudadanos. La existencia o ausencia de intervenciones sociales ilustran los desafíos que hoy surgen para el quehacer profesional y ponen de manifiesto la necesidad de repensar las premisas fundamentales sobre las que se ha construido el Trabajo Social. Esta transformación del estado de bienestar implica repensar el significado mismo de lo social.

\section{Un Estado de bienestar en proceso de transformación}

A nivel internacional el estado de bienestar sueco llegó a representar un modelo a imitar para muchos países. La ideología que avaló las reformas sociales fue capturada en la metáfora «hogar del pueblo" (folkhemmet) que creó una analogía entre hogar y sociedad para capturar 
la racionalidad sobre la que se construiría el bienestar ciudadano (Hirdman, Björkman \& Lundberg, 2012). Esta metáfora daba por sentado que la población sueca era una población cultural y étnicamente homogénea. Durante mucho tiempo la imagen de la sociedad de bienestar sueca era la de una sociedad que aspiraba a la inclusión de todos sus ciudadanos, si bien estudios recientes muestran que también dentro de este proyecto de bienestar supuestamente incluyente, siguieron existiendo diferentes formas de exclusión social (Montesino, 2012; Runcis, 1998; Tydén, 2002).

En términos generales la mayoría de los investigadores sociales coinciden en que durante el período de auge del estado de bienestar los derechos sociales se hicieron extensivos a toda la población laboralmente activa, un desarrollo que permitió la estabilidad económica gracias al pleno empleo, situación única favorecida por la expansión económica que perduró hasta principios de 1970. Suecia pasó a ser entonces identificada internacionalmente como el estado de bienestar por excelencia, conceptualizado como el Modelo Socialdemócrata del Estado de Bienestar (Esping-Andersen, 1990). En el caso del Trabajo Social este mismo desarrollo se expresó en el rol activo del estado en la determinación de los contenidos de las intervenciones sociales, en la responsabilidad pública sobre el financiamiento de esas intervenciones y en la organización pública del Trabajo Social.

Las condiciones que permitieron ese desarrollo histórico han cambiado. Se trata de una ruptura que cuestiona la autoimagen que los suecos mantuvieron durante largo tiempo sobre la organización y las condiciones de su propio bienestar social. Esta ruptura involucra temas tan fundamentales como el acceso a los derechos sociales, la igualdad social, la situación laboral, las oportunidades de movilidad social, el rendimiento escolar, etc. (OECD, 2008, 2011, 2013). Se trata, como antes mencionábamos, de reestructuraciones que están ocurriendo en toda Europa, pero que presentan características diferentes de acuerdo a los modelos de bienestar social desarrollados en cada país (Lorenz, 2006). En el caso de Suecia con su imagen internacional de un estado de bienestar progresista e igualitario es necesario describir estas transformaciones y discutir las repercusiones que estos cambios tienen sobre las formas de entender y hacer el Trabajo Social. La globalización de la economía y el consecuente cuestionamiento de las fronteras nacionales también obligan a repensar las premisas sobre la que se construyó la supuesta universalidad de los derechos sociales.

Aunque no es nuestra intención discutir las causas de esas transformaciones, es necesario señalar que se trata de cambios cuyas causas y consecuencias son múltiples y complejas (Hartman, 2011). La crisis económica que empezó en la década del 90 afectó a Suecia, al igual que a otros países. La piedra angular del modelo de bienestar sueco (el pleno empleo) se rompió cuando empezó a aumentar la desocupación, del 1,7\% al 8,3\% en la década de 1990. Hoy, al igual que en otros países europeos, la desocupación afecta sobre todo a los jóvenes entre los cuales la tasa de desempleo asciende hoy al $24 \%$. En esta estadística constatamos la sobrerrepresentación de los inmigrantes (SCB, 2013; Socialstyrelsen, 2010). En las comparaciones internacionales Suecia ha perdido su posición de liderazgo, un estudio reciente muestra que Suecia descendió del primer lugar al lugar número catorce en la clasificación sobre desigualdad de ingresos y pobreza (OECD, 2013).

Suecia ha dejado de ser el país caracterizado por la igualdad social, aunque la infraestructura construida anteriormente (por ejemplo el sistema de seguros sociales) aún sigue teniendo efectos compensatorios para la mayoría de los ciudadanos. Pero también surgen problemas desconocidos para la generación que vivió la época de auge del estado de bienestar. Por ejemplo aumentan las cifras de quienes van quedando excluidos del acceso a beneficios sociales; crecen los grupos que no tienen ni nunca han tenido acceso a esos beneficios; diferentes estudios indican que jóvenes y migrantes están también sobrerrepresentados en la estadística (Socialstyrelsen, 2010). Las políticas de austeridad pública son un resultado de la aplicación de una política neoliberal en la reorganización de los Servicios de Bienestar inspirada en los principios de la Nueva Gestión Pública.

Las consecuencias de esta reestructuración han creado, entre otras cosas, un clima de mayor inseguridad respecto al futuro, una situación desconocida para la mayor parte de los 
suecos. Este clima de inseguridad ha dejado espacio para el surgimiento de manifestaciones abiertamente racistas o/y xenófobas, problema que afecta directamente la vida cotidiana de los migrantes y que también se expresa en cierto consenso respecto a las políticas de inmigración que son cada vez más restrictivas. Aquí es necesario señalar que la composición de la población sueca ha cambiado. Suecia cuenta con una población de más de 9,5 millones de habitantes, el $10 \%$ de esa población es de origen extranjero (SCB 2013). La diversidad cultural y étnica es una característica de la población sueca hoy en día. Otra característica que es necesario tomar en cuenta es el creciente envejecimiento de la población, un $19 \%$ de la población tiene más de 65 años. Las expectativas de vida son para las mujeres de 84 años y para los hombres de 80 (SCB 2013). Es decir, aumenta la cantidad de individuos potencialmente dependientes de los Servicios de Bienestar, servicios que tienden a orientarse cada vez más a estrategias de intervención basadas en las relaciones de mercado (Hartman, 2011). En este artículo discutimos la complejidad de los cambios en curso desde el área de protección de la infancia, específicamente el Trabajo Social con niños migrantes que llegan a Suecia sin compañía adulta.

\section{Trabajo Social, infancia y niños migrantes}

La estrecha relación entre estado y el Trabajo Social que ha existido en Suecia está cambiando sus contenidos y sus formas, lo cual se ha expresado, entre otras cosas, en la introducción de un lenguaje administrativo que refleja una nueva comprensión de lo social. Esta transformación involucra diferentes aspectos del Trabajo Social y se lleva a cabo a diferentes niveles. A nivel estatal la responsabilidad sobre el bienestar social se está definiendo en términos individuales, cada individuo es considerado responsable de su propio bienestar. El área de bienestar se reestructura en organizaciones donde la gestión de presupuestos y el liderazgo administrativo transforman los contenidos del trabajo profesional (Berg, Barry \& Chandler, 2008). En este paisaje las nuevas prácticas evalúan situaciones individuales y los nuevos métodos de intervención se basan en criterios de efectividad, criterios que cambian la relación tradicional entre trabajadores sociales y clientes. Se trata de transformaciones que tienen implicaciones para la identidad profesional, las formas de conocimiento que legitiman las intervenciones sociales y las prácticas de trabajo de los trabajadores sociales (Bergmark, Bergmark \& Lundström, 2012). Nuestro análisis del Trabajo Social con niños migrantes se concentra en ciertas prácticas de los sistemas de protección de la infancia: la atención individual a niños migrantes en los servicios de bienestar social y la supuesta protección institucional a la que pueden llegar a tener derecho esos niños.

El Trabajo Social ha sido una actividad basada en representaciones sobre una sociedad cultural y étnicamente homogénea, donde los derechos sociales alcanzan a todos los ciudadanos y la familia nuclear es considerada la institución ideal para la protección del niño. La situación de los niños migrantes permite identificar los límites de esas representaciones y cuestiona la postulada universalidad en el acceso a los derechos sociales tanto hoy como en otros períodos de la historia del estado de bienestar.

La protección de la infancia se constituyó históricamente como el área clásica para la intervención de los trabajadores sociales, una protección construida sobre discursos legitimados por principios de solidaridad colectiva (Jordan, 2005). Esos mismos discursos contribuyeron al establecimiento de representaciones que aún se mantienen, pero que en las respuestas a la problemática de los niños migrantes, muestran fuertes contradicciones que conducen a repensar las premisas sobre las que se constituyeron las representaciones sobre la infancia (dependencia y necesidad de protección) y la organización territorial de los sistemas de protección de los niños (Jiménez, 2011). Se trata de sistemas construidos para atender exclusivamente a niños con estatus jurídico de residentes. La presencia de los niños migrantes pone de manifiesto los límites de los principios básicos de pertenencia a la organización social.

En Suecia las políticas de bienestar infantil están centradas alrededor de la familia, su objetivo es preservar la cohesión familiar y evitar al máximo la separación del niño de su grupo familiar (Höjer \& Forkby, 2011). Las teorías y prácticas de las que dispone el profesional han 
sido articuladas de acuerdo a principios generales de prevención cuyo fin es el mantenimiento, reforzamiento $\mathrm{o} / \mathrm{y}$ reconstrucción de los lazos familiares para la protección del niño (Ellison, 2007). Las leyes que orientan el Trabajo Social con niños están también inspiradas por nuevas concepciones sobre la infancia que reconocen al niño como sujeto de derechos cuya agencia debe ser interpretada de acuerdo a la racionalidad de quien actúa. El enfoque familiar de los servicios de bienestar ha sido criticado porque tiende a descuidar la perspectiva del niño en la construcción de medidas para mejorar su situación, esa crítica inspira hoy la creación de algunas actividades que tratan de regular situaciones familiares complejas desde la perspectiva del niño (violencia familiar, abuso de drogas, etc.), actividades destinadas a dar apoyo a niños en situación de vulnerabilidad que tienen estatus de residentes en Suecia.

La regulación estatal establecida en la legislación sobre el Servicio de Bienestar Social ha dejado cierto espacio para la acción profesional, denominado como espacio discrecional (Svensson, Johnsson \& Laanemets, 2008). La cuestión es de qué manera ese espacio se mantiene o reduce cuando los Servicios de Bienestar se reorganizan desde nuevas racionalidades y cuando los trabajadores sociales enfrentan situaciones que están fuera de los marcos establecidos.

Las migraciones actuales de niños son objeto de constantes controversias políticas en Suecia. La construcción polarizada de discursos sobre los niños migrantes debe entenderse dentro de los marcos de las políticas de inmigración cada vez más restrictivas que se implementan en los países de Europa del norte (Eastmond, 2011). La migración de niños tiene una larga historia (Jiménez, 2011; Socialstyrelsen, 2013). Hasta fines del siglo XX la situación legal y social de los niños migrantes era tratada en Suecia como parte de una problemática situación familiar. Ante la ausencia de la familia era tarea de los trabajadores sociales incorporar a esos niños a una institución que funcionara como sustituto de la institución familiar. Son estas mismas estrategias las que estructuran hoy el quehacer de los trabajadores sociales. El problema es el desfase que existe entre esas construcciones y las diversas situaciones que enfrentan los trabajadores sociales en su trabajo cotidiano. La problemática de los niños que migran solos es testigo de esa complejidad.

De acuerdo a la estadística oficial aumenta la cantidad de niños migrantes que llegan solos a Suecia. El año 2004 fueron 388 niños menores de 18 años los que pidieron asilo en Suecia y esta cifra ha seguido aumentando. De acuerdo con el Departamento de migraciones, este cambio se empezó a notar a partir del año 2006, durante el cual fueron 816 los solicitantes de asilo, 1510 el año 2008, dos años más tarde la cifra aumentó a 2393 y en el año 2012 fueron 3578 los niños solicitantes de asilo. La mayoría de esos niños tienen entre 12 y 18 años (Migrationsverket, 2013).

La atención a los niños migrantes está condicionada por diferentes leyes (leyes que regulan las migraciones y el asilo político, leyes que regulan los servicios de bienestar social y leyes que regulan los derechos del niño) (Socialstyrelsen, 2013).

Las autoridades suecas distinguen, de acuerdo a la situación jurídica y social, tres categorías de niños migrantes: los que están en situación de espera (solicitantes de asilo), niños que ya han obtenido el asilo y que están siendo incorporados a los sistemas tradicionales de protección para niños y niños a los que se les ha negado el asilo (incluidos en la categoría sin papeles). Es responsabilidad de las autoridades municipales, y en concreto de los Servicios de Bienestar Social, organizar la acogida de los niños en situación de espera y también la de los niños que han obtenido el asilo político. La atención a las dos primeras categorías está basada en los principios generales formulados en la Ley de Servicios Sociales, en concreto significa que es responsabilidad de los servicios de bienestar municipales otorgar vivienda, tutoría y escolarización a estos migrantes (Migrationsverket, 2013). Los niños a los que se les ha negado el asilo quedan jurídicamente excluidos de los sistemas de protección (Björngren-Cuadra \& Staaf, 2012). No existen cifras exactas sobre cuántos son los migrantes que viven en situación irregular en Suecia. Pero el Departamento de Asuntos Sociales (Socialstyrelsen) especula que alcanzan a una cifra de entre 10 mil y 50 mil, de los cuales al menos 3000 serían niños, según un informe publicado el año 2010 (Socialstyrelsen, 2010). Como an- 
tes señalábamos la cifra de niños que llegan solos a Suecia ha aumentado, como también aumentan las cifras de migrantes a los que se les niega el asilo y que se quedan en Suecia. Entre ellos hay también niños que viven escondidos con sus familias.

\section{Atención individual}

La situación de los niños que están obligados a vivir en la clandestinidad es preocupante, según sostiene un informe más reciente (Socialstyrelsen, 2013). Trabajadores sociales empleados en los Servicios de protección de la infancia son testigos de que crece el número de niños que piden apoyo económico o asistencia especial en sus unidades. Un estudio publicado recientemente muestra que las respuestas de los funcionarios que trabajan en esas unidades son diversas (Björngren-Cuadra \& Staaf, 2012). Los trabajadores sociales se apoyan en diferentes leyes para justificar sus decisiones. Algunos remiten a las leyes de control de la Inmigración explicando que estos niños carecen de permiso de residencia y que no pueden estar incluidos en los programas de asistencia económica. Dentro de esta categoría encontramos trabajadores sociales que derivan a los niños a organizaciones no gubernamentales y trabajadores sociales que niegan la ayuda a esos niños argumentando que no tienen derecho a la asistencia económica porque carecen de permiso de residencia y no están bajo la tutela de su municipio. Aparte de esas respuestas el mismo estudio identifica otras formas de resolver la situación. En los servicios de bienestar encontramos también trabajadores sociales que acogen a los solicitantes incluyéndoles en casos de atención urgente, se trata de trabajadores sociales que otorgan asistencia a los niños y que legitiman su decisión en la misma Ley de Servicios de Bienestar. Como antes señalábamos esta ley otorga al profesional un espacio moderado para tomar decisiones de acuerdo a los propios criterios profesionales. Björngren-Cuadra \& Staff (2012) concluyen que este espacio discrecional puede ser usado tanto para excluir como para incluir a los niños en los Servicios de Bienestar Social. Los trabajadores sociales que incluyen a los niños se apoyan en la Ley de Servicios de bienestar, los que deciden excluirles se apoyan en la Ley de control de la inmigración (ibíd.). Aparte de es- tas respuestas que están dentro de los marcos institucionalizados del bienestar social, podemos constatar que algunos trabajadores sociales también crean y participan en otros espacios donde trabajan de acuerdo a las perspectivas de quienes sufren los efectos de las políticas gubernamentales, es decir, que pertenecen al activismo social.

Profesionales de diferentes especialidades se han organizado para denunciar la situación de los migrantes sin papeles, especialmente la situación de los niños que ha contribuido a crear espacios de activismo social, espacios nuevos en un paisaje extremadamente regulado como ha sido y aún es el paisaje social sueco. Se trata de actores que desde diferentes organizaciones no gubernamentales tratan de otorgar orientación jurídica y social a los migrantes sin papeles y que también tratan de influir en las decisiones de las autoridades denunciando la situación en que se encuentran. Muchos de estos activistas son jóvenes estudiantes universitarios, otros son profesionales que trabajan en los servicios sociales, educacionales y de salud pública. Se trata de profesionales que se niegan a aceptar los límites establecidos por sus propias organizaciones y que cuestionan los costos humanos creados por las formas actuales de regulación social. En algunos municipios la acción de estos grupos ha contribuido a cambiar ciertas directivas locales. Por ejemplo en la región de Escania, al sur de Suecia, los niños sin papeles tienen derecho a asistir a la escuela y también a recibir atención médica de urgencia. Estas posibilidades están siendo socavadas por una nueva directiva cuyo objetivo es la expulsión de los migrantes en situación irregular. Esta directiva criminaliza directamente a los migrantes sin papeles y autoriza a la policía para averiguar el paradero de estos niños y encarcelarles hasta que sean expulsados del país. Este es un proyecto financiado por el Fondo de Repatriación de la comunidad europea cuyo lenguaje reduce y entiende la intervención como un trámite administrativo «gestionamiento de casos de forma eficiente y efectiva, cuyo fin es la ejecución jurídicamente segura de una sentencia...» (Migrationsverket, 2013). Llama la atención el hecho de que los niños buscados por la policía son los que carecen de papeles y, sin embargo, la actitud frente a la situación de 
aquellos niños que diariamente desaparecen de las instituciones donde son internados para su supuesta protección es otra.

\section{Hogares de acogida}

La presencia de niños migrantes justifica el crecimiento de ciertas formas institucionalizadas de intervención. Espacios que independientemente de los discursos que les legitiman contribuyen a la formación y expansión de «geografías de la espera», lugares donde transcurren las vidas de quienes están a la espera de que se resuelva su situación jurídica, «lugares de tránsito» o lugares que justifican su existencia desde las políticas de las sociedades que les acogen (Correa, Bartolotto \& Musset, 2013). En el caso de los niños migrantes se crean instituciones que supuestamente protegen y orientan a esos niños en su «tránsito» por Suecia o en su «entrada a la sociedad». En esos lugares se crea una cotidianeidad que imita las rutinas de la vida familiar. Se trata de instituciones que tienen una historia que se inició a fines del siglo XIX para internar niños con diferentes problemáticas clasificadas como anormales (Sallnäs, 2000). En el lenguaje de los sistemas de bienestar estas instituciones son descritas como hogar sustituto. Tradicionalmente estos hogares han acogido niños con problemas de agresividad, drogadicción o delincuencia; generalmente niños con situaciones familiares complejas. Hoy existen 837 Hogares de acogida para niños que están registrados y autorizados por la Dirección Nacional de Asistencia Institucional (StatensInstitutionsstyrelse, 2013). Las Hogares de acogida para niños migrantes se han multiplicado durante los últimos años. Hoy en día estos alcanzan los 199. La responsabilidad de estas instituciones les corresponde a las autoridades municipales.

Los procesos de privatización del área social se reflejan claramente en el traspaso de estas instituciones al área privada (Sallnäs, 2005). No existen aún estudios sobre el significado que esta privatización tiene para los Hogares de acogida de niños migrantes, aunque si existen denuncias sobre abusos de poder y elevados presupuestos que sobrepasan los acuerdos iniciales. Diferentes empresas venden sus servicios a los municipios que son hoy los responsables de organizar el cuidado de los niños que llegan a Suecia sin sus familias, tanto los que están solicitando asilo como los que ya lo han obtenido. El empleo de trabajadores sociales en estas empresas se hace a través de la subcontratación. Las consecuencias que estas formas de empleo tienen sobre la autonomía profesional es una temática que todavía está por estudiarse.

Los Hogares de acogida están sometidos a controles generales aplicados a todas las instituciones que atienden a menores de edad. La desaparición de niños migrantes de los Hogares de acogida ha sido objeto de un estudio recientemente publicado en Suecia (Mikkelsen \& Wagner, 2013). Según este informe, entre el año 2007 y el año 2012, han desaparecido 783 niños de estos hogares de acogida. Este fenómeno ha sido descrito en un estudio comparativo internacional donde se sostiene que la desaparición de niños no es un fenómeno excepcional ni marginal sino que existe y aumenta cada día en diferentes países de Europa (Hedjam, 2009). El caso sueco confirma esas afirmaciones. La actitud de las autoridades suecas respecto a esta situación es pasiva, el personal que trabaja a diario con esos niños registra esas ausencias en sus informes administrativos. Cuando los niños se van o desparecen son registrados como niños que voluntariamente «abandonan» el hogar y dejan de ser considerados responsabilidad de esas instituciones. La policía declara que detrás de esas desapariciones no han identificado actividades criminales y que no tienen motivos para seguir el rastro de esos niños, una actitud totalmente opuesta a la que la policía asume cuando desaparece un niño sueco (Mikkelsen \& Wagner, 2013).

Las rutinas creadas en los Hogares de acogida no parecen garantizar la protección de los niños. Los niños desaparecen de esas instituciones sin dejar rastro alguno. Según el estudio antes mencionado los niños que desaparecen son aquellos cuyas solicitudes de asilo han sido denegadas (ibíd.). La desaparición de esos niños habla de la distancia existente entre las formas de protección ofrecidas por los servicios de bienestar y las vidas de los niños migrantes.

Otro aspecto a considerar es la vida cotidiana que se organiza en torno a los niños migrantes en estos lugares. Un estudio publicado recientemente describe la relación de los niños mi- 
grantes a los que se les ha otorgado asilo con el personal de los Hogares de acogida. Este estudio se basa en entrevistas y describe la percepción positiva que tienen los niños del personal, percepción opuesta a la que los mismos niños tienen de quienes administraron su petición de asilo (Lundberg, 2012). En otro estudio que se está llevando a cabo en el sur de Suecia, los menores migrantes expresan la distancia que existe entre sus propias experiencias de vida, sus conocimientos y el espacio para expresar y utilizar esos conocimientos y experiencias en la vida que las autoridades suecas han organizado para ellos (Montesino \& Hansson, 2013). Los entrevistados describen además situaciones difíciles que no pueden comunicar al personal porque estos no están en condiciones de comprender sus problemas (ibíd.). Las complejas problemáticas de los niños llevan a los trabajadores sociales a interpretar sus vidas de acuerdo a representaciones tradicionales (niños dependientes, víctimas, etc.) dificultando la incorporación de las experiencias, conocimientos y estrategias de bienestar desarrolladas por estos niños durante el proceso migratorio. El personal de esas instituciones carece de herramientas de análisis que les permita entender esas problemáticas, sus percepciones siguen elaborándose dentro de los marcos nacionales de integración social. Los conocimientos, vivencias y relaciones de los niños migrantes se desarrollan en espacios transnacionales. Espacios que siguen formando parte de la vida cotidiana de los niños después de que llegan a Suecia.

\section{Conclusiones}

Suecia es un estado de bienestar que está llevando a cabo transformaciones radicales tanto de su organización como de su forma de entender y abordar los problemas sociales. Al mismo tiempo se puede constatar que la composición de la población demanda respuestas plurales que se adecúen a la diversidad de problemáticas sociales. Estudios recientes identifican diferentes respuestas. Algunos estudios sostienen que los trabajadores sociales se subordinan a las nuevas formas de abordar los problemas sociales elaborados desde la Nueva Gestión Social. Otras respuestas, sin ser parte de una alternativa expresamente formulada, usan los espacios disponibles dentro del marco de leyes establecidas para dar asistencia a los que la solicitan independientemente de su situación jurídica. Aparte de esas respuestas han surgido durante los últimos años formas de activismo social periféricas pero que están presentes en el debate público y que denuncian la situación de los niños migrantes y exigen su inclusión en los sistemas de protección social.

La llegada de los niños migrantes representa un desafío a las concepciones de lo social y cuestiona las formas institucionalizadas creadas para resolver los problemas sociales. Lo social fue construido como un espacio territorialmente limitado por las fronteras del estado nacional, los grupos e individuos incluidos en las intervenciones sociales eran aquellos que formaban parte de esa unidad territorial. Las migraciones globales han cambiado los contenidos de lo social. En el caso sueco este cambio se refleja, entre otras cosas, en la diversidad cultural y étnica de la población. El Trabajo Social trata de adaptarse a este paisaje social desde el paradigma de la integración, una integración concebida desde instituciones construidas para atender a una población homogénea. El caso de los niños migrantes hace manifiestas esas y otras contradicciones. Siendo la protección de la infancia una de las áreas que han legitimado la existencia misma del Trabajo Social, nos encontramos ante una situación donde las instituciones que otorgan esa protección excluyen a niños migrantes o ignoran el destino de esos niños reduciendo sus prácticas a rutinas exclusivamente administrativas. Paralelo a este desarrollo también podemos identificar cierta creatividad en las acciones de grupos que cuestionan y discuten alternativas que correspondan a las realidades que sufren quienes han quedado fuera de los sistemas de protección social.

\section{Referencias bibliográficas}

Berg, E., Barry, J., \& Chandler, J. (2008). New public management and social work in Sweden and England. International Journal of Sociology and Social Policy, 28(3/ ), 114-128.

Bergmark, A., Bergmark, A., \& Lundström, T. (2012). The mismatch between the map and the terrain. Evidence-based social work in Sweden. European Journal of Social Work, 15(4), 598-609. 
Björngren-Cuadra, C., \& Staaf, A. (2012). Public Social Services'encounters with irregular migrants in Sweden: amid values of social work and control of migration. European Journal of Social Work, 1-16. doi: 10.1080/13691457.2012.739556

Correa, V., Bartolotto, I., \& Musset, A. (2013). Geografias de la espera. Migrar, habitar y trabajar en la ciudad de Santiago (Chile), 1990-2012. Santiago de Chile: Uqbar Editores.

Eastmond, M. A., H. (2011). In the Best Interest of the Child? The Politics of Vulnerability and Negotiations for Asylum in Sweden. Journal of Ethnic and Migration Studies, 37(8), $1185-$ 1200.

Ellison, M. (2007). Contested terrains within the neo-liberal project. Equal Opportunities International, 26(4), 331-351.

Esping-Andersen, G. (1990). The three worlds of welfare capitalism. Cambridge: Polity.

Hartman, L. (2011). Konkurrensens konsekvenser : vad händer med svensk välfärd. Stockholm: SNS förlag.

Hedjam, S. (2009). Disparitions, départs volontaires, fugues. Des enfants de trop en Europe?

Hirdman, Y., Björkman, J., \& Lundberg, U. (2012). Sveriges historia. 1920-1965 : [rösträtt och demokrati, fackföreningar, förbudsomröstning, depression, Adalskravaller, Kreugerkrasch, kohandel, Saltsjöbadsavtal, folkhem, krispolitik, urbanisering, neutralitet, samlingsregering, beredskapstid, ATP-reform, radio och teve, bilism, rekordår]. Stockholm: Norstedt.

Höjer, S., \& Forkby, T. (2011). Care for Sale: The Influence of New Public Management in Child Protection in Sweden. British Journal of Social Work, 41, 93-110.

Jiménez, M. (2011). Intrusos en la fortaleza. Menores marroquies migrantes en la Frontera Sur de Europa. Madrid: Facultad de Filosofía y Letras, Universidad Autónoma de Madrid.

Jordan, B. (2005). Sex, pengar och makt : om det kollektiva livets omvandlingar. Göteborg: Daidalos.

Lorenz, W. (2006). Perspectives on European social work: from the birth of the nation state to the impact of globalisation. Opladen: Budrich.

Lundberg, A. D., Lisa. (2012). Unaccompanied Children Seeking Asylum In Sweden: Living Conditions From A Child-Centred Perspective. Refugee Survey Quarterly, 31(2), 54-75. doi: 10.1093/RSQ/HDS003

Migrationsverket. (2013). Ett gemensamt ansvar för ensamkommande barn och ungdomar. Suecia: www.migrationsverket.se.

Mikkelsen, J., \& Wagner, K. (2013). De förlorade barnen : ett reportage. Stockholm: Natur \& kultur.

Montesino, N. (2012). Social Disability: Roma and Refugees in Swedish Welfare Journal of Migration, Health and Social Care, 8(3).

Montesino, N., \& Hansson, V. (2013). Ensamkommande barnmigranter. Comunicación presentda en el IMISCOE, Malmö, Suecia.

OECD. (2008). Growing unequal? : income distribution and poverty in OECD countries. París: Organisation for Economic Co-operation and Development.

OECD. (2011). Divided we stand : why inequality keeps rising. París: OECD Publishing.

OECD. (2013). Crisis squeezes income and puts pressure on inquality and poverty. In OECD (Ed.). París: OECD.

Runcis, M. (1998). Steriliseringar i folkhemmet. Stockholm: Ordfront.

Sallnäs, M. (2000). Barnavårdens institutioner : framväxt, ideologi och struktur. Stockholm: Univ.

Sallnäs, M. (2005). Vårdmarknad med svårigheter- om privata aktörer inom institutionsvården för barn och ungdomar. Socialvetenskaplig tidskrift, 2-3, 226-245.

SCB. (2013). Statistisk centralbyrå. Retrieved 20130701, 2013

Socialstyrelsen. (2010). Social rapport. Stockholm: Socialstyrelsen.

Socialstyrelsen. (2013). Socialtjänstensarbete med ensamkommande barn och ungdomar, en vägledning. Stockholm: Socialstyrelsen.

StatensInstitutionsstyrelse. (2013). Faktabank. Retrieved 20130614 
Sunesson, S. (2003). Socialt arbete - en bakgrund till ett forskningsämne. In -. X. R. Högskoleverkets rapportserie (Ed.), Socialt arbete : en nationell genomlysning av ämnet. Stockholm: Högskoleverket.

Svensson, K., Johnsson, E., \& Laanemets, L. (2008). Handlingsutrymme : utmaningar i socialt arbete. Stockholm: Natur \& kultur.

Tydén, M. (2002). Från politik till praktik : de svenska steriliseringslagarna 1935-1975. Stockholm: Almqvist \& Wiksell International. 